Abstracta Iranica $\begin{aligned} & \text { Abstracta Iranica } \\ & \text { Revue bibliographique pour le domaine irano-aryen } \\ & \text { Volume 42-43| 2021 } \\ & \text { Comptes rendus des publications de 2019-2020 }\end{aligned}$
Vladimir D. Kuznetsov. "The Cimmerian Bosporus
in the 5th Century BC (an Old Persian Inscription
from Phanagoria)"

Rémy Boucharlat

(2) OpenEdition

Journals

Édition électronique

URL : https://journals.openedition.org/abstractairanica/52522

DOI : 10.4000/abstractairanica.52522

ISSN : 1961-960X

Éditeur :

CNRS (UMR 7528 Mondes iraniens et indiens), Éditions de l'IFRI

Référence électronique

Rémy Boucharlat, « Vladimir D. Kuznetsov. "The Cimmerian Bosporus in the 5th Century BC (an Old Persian Inscription from Phanagoria)" », Abstracta Iranica [En ligne], Volume 42-43 | 2021, document 33, mis en ligne le 15 avril 2021, consulté le 15 décembre 2022. URL : http://journals.openedition.org/ abstractairanica/52522 ; DOI : https://doi.org/10.4000/abstractairanica.52522

Ce document a été généré automatiquement le 15 décembre 2022.

Tous droits réservés 


\title{
Vladimir D. Kuznetsov. "The Cimmerian Bosporus in the 5th Century BC (an Old Persian Inscription from Phanagoria)"
}

\author{
Rémy Boucharlat
}

\section{RÉFÉRENCE}

Vladimir D. Kuznetsov. "The Cimmerian Bosporus in the 5th Century BC (an Old Persian Inscription from Phanagoria)", ACSS 25, 2019, p. 8-43.

1 Dans cet article paru à l'origine en russe en 2017, l'A. propose trois hypothèses pour expliquer la présence de ce fragment de stèle royale en vieux-perse citant le nom de Darius I sur le rivage nord de la mer Noire. Pour le contexte archéologique de la découverte voir Vladimir D. Kuznetsov, Alexander B. Nikitin. "An old Persian Inscription from Phanagoria", ACSS 25, 2019, p. 1-7 (voir le compte rendu dans ce numéro).

2 1. Il s'agirait d'une partie d'une inscription laissée lors de la campagne de Darius contre les Scythes d'Europe puis rapportée à Phanagoria comme un élément parmi d'autres de ballast d'un bateau venu d'un port d'Ionie.

3 2. Elle aurait été laissée dans la ville lors de la campagne d'Ariaramnès, satrape de Cappadoce, contre les Scythes en 515-514, sur laquelle l'A s'étend, insistant sur l'abondance des sceaux-cylindres de style achéménide provenant de la région nord de la mer Noire et s'interrogeant sur la réalité de l'occupation de la région.

43 . Avec des arguments fondés sur les sources classiques, l'A. pencherait plutôt pour la reconstitution suivante : l'érection de la stèle sur ordre de Xerxès, après la capture de la cité par les troupes perses dans le contexte des guerres gréco-perses, dites 
«médiques »; la mise en place d'un roi dépendant du Perse ; plus tard, la destruction de la ville lors de l'expédition de la flotte athénienne de Périclès vers 438-437.

5 Cette hypothèse d'une stèle érigée localement est rejetée aussi bien par $E$. Rung et 0. Gabelko (Eduard Rung, Oleg Gabelko. "From Bosporus ... to Bosporus: A New Interpretation and Historical Context of the Old Persian Inscription from Phanagoria", Iranica Antiqua, 54, 2019, p. 83-125 : voir le compte rendu dans ce numéro) ainsi que E. Shavarebi (Ehsan shavarebi. "An Inscription of Darius I from Phanagoria (DFa): Preliminary report of a work in progress", ARTA, 2019.005, 2019, 15p. : voir le compte rendu dans ce même numéro) qui voient l'origine de la stèle dans le Bosphore anatolien, puis le transport de l'élément retrouvé à Phanagoria.

\section{AUTEURS}

\section{RÉMY BOUCHARLAT}

UMR 5133 CNRS-Université de Lyon 